\title{
Influence of Sun-View Geometries on the Relationships Among Vegetation Indices, LAI, and Absorbed PAR
}

\author{
José C.N. Epiphanio ${ }^{1}$ Alfredo R. Huete, and Hui Qing Liu
Dept. of Soil and Water Science, University of Arizona, 429 Shantz Bldg. \#38, Tucson, Arizona U.S.A. 85721 \\ Tel: 602-621-3228 FAX 602-621-1647 email: epiphani@ccit.arizona.edu \\ IVisiting Scholar, INPE, 12201 - São José Campos, SP, Brazil
}

The absorbed photosynthetically active radiation (APAR) and leaf area index (LAI) of vegetated surfaces have been addressed as important parameters to be included in growth and productivity models. However, surface heterogeneities render the signal detected by the remote sensor sensitive to illumination/view geometry and background (e.g. soil, litter) reflectance properties. In addition, there is the influence of the intervening and variable atmosphere. In this study, the Myneni radiative canopy model in conjunction with the ' $6 S^{\prime}$ ' atmospheric model, were used to simulate the optical properties of both uniform and heterogeneous vegetated surfaces. The relationships among fAPAR, LAI, and VIs under various sunview geometries are investigated.

\section{INTRODUCTION}

Remote sensing can provide information about targets through measurements of radiation. Visible radiation that interacts with plant canopies is referred as photosynthetic active radiation (PAR). This radiation is used by the plants to transform water and $\mathrm{CO}_{2}$ into organic compounds, e.g. carbohydrates. The rate at which this transformation occurs depends on many factors, including incident radiation, crop species, plant stress, and canopy capability to intercept light. The fraction of intercepted light absorbed by a canopy is referred as absorbed photosynthetically active radiation or fAPAR. This fraction expresses the part of the total incident radiation that is effectively available to the plant to be used in photochemical processes. Many studies have demonstrated that this fraction can be estimated by remote sensing measurements (Daughtry et al., 1992). Another important vegetation parameter is the leaf area index (LAI), which has been the subject of many studies in remote sensing.

Remote sensing in the optical region deals mainly with the fraction of radiation reflected by the canopies. To derive relationships between reflected radiation and physical canopy parameters, two or more bands are used in the form of vegetation indices (VI). The most extensively used combination of bands to monitor vegetation is the normalized difference vegetation index (NDVI). Recently other modifications have been introduced to the NDVI to reduce noise influences, like soil (Huete, 1988) and atmospheric effects (Kaufman and Tanré, 1992).

The interactions among soil, canopy and atmosphere are complex. Each of these components has optical properties that vary spatially and temporally. In addition there is the sensor/illumination geometry that affects these coupled signals. To study these complex interactions and their impact on the relationships between vegetation and remote sensing parameters models are often used (Goward and Huemmrich, 1992).

As the new satellites on the Earth Observing System (EOS) are going to be launched, it is important that the factors influencing the reflectance and VIs and their relationships to physical target parameters be understood. In this study, we use a radiative transfer model to simulate different canopies and analyze VI relationships to fAPAR and LAI. The variables analyzed are view angle, soil background and atmosphere turbidity.

\section{METHODS}

The 3-dimensional Myneni canopy model was used to simulate directional canopy reflectances (Myneni et al., 1992). This model assumes a finite, horizontal, and extensive 3-D field, where the incident radiation in the given direction, $\Omega^{\prime}\left(\mu^{\prime}, \varphi^{\prime}\right)$, reaches the canopy uniformly in one direction and is scattered in a given direction, $\boldsymbol{\Omega}(\mu, \varphi)$, with azimuthal angle $\varphi$, polar angle $\theta=\cos ^{-1}(\mu)$ with respect to the outward normal, and $\mu$ is the direction of the photon travel. The canopy is divided into small tridimensional cells and the radiative transfer function is solved for the entire grid. The model predicts the radiation absorbed by the canopy, by the soil, the albedo, and the emerging radiation from the canopy in all directions, thus allowing the derivation of the reflectance in different view angles, including the hot spot. The inputs are the dimensions of the stand $(x, y, z)$, ground cover (GC), leaf area index of the clump (CLAI) and dimensions of the clump, leaf angle distribution, leaf reflectance and transmittance, soil albedo, and illumination geometry (zenith and azimuth).

For this study, the soil albedo was allowed to vary in 0.05 steps, from 0.10 to 0.35 ; the ground covers (GC) were $0.25,0.50$, 0.75 and 0.99 for two levels of clump LAI (CLAI $=2$ and 4 ). The simulations were done in the principal plane, with the zenith view angle varying from $-70^{\circ}$ (antisolar viewing direction) to $+70^{\circ}$ (forward scattering direction) in $10^{\circ}$ steps, and the sun was positioned at $180^{\circ}$ (azimuth) and $40^{\circ}$ (zenith). Three levels of leaf reflectance/transmittance were used, $0.05 / 0.05,0.48 / 0.48$, and $0.097 / 0.035$ for the red, near infrared (NIR), and visible, respectively. The values for red and NIR are average values from the literature, while visible values were simulated from Jacquemoud and Baret (1990). The fAPAR values were derived from values for the visible band, instead of using the red band, in order to have a more realistic evaluation of fAPAR.

The evaluation of the atmospheric effect on reflectances and VI's was done by using the $6 \mathrm{~S}$ model. The conditions assumed were at satellite level, with a U.S. standard atmosphere, 62 , continental aerosol, with $23 \mathrm{~km}$ visibility, using the MODIS bands $(0.620-0.670 \mu \mathrm{m}$ and $0.841-0.876 \mu \mathrm{m}$ for red and NIR, respectively), and $0.4-0.7 \mu \mathrm{m}$ for the visible band. The surface was considered homogeneous, and the model was run for each view angle direction simulated in the Myneni canopy model. In this study, we tested the NDVI, the soil-adjusted vegetation index (SAVI), and the simple ratio (SR). The soil NIR reflectance was taken as 0.05 higher than the red reflectance, following the approximate regression of red $v s$. NIR soil reflectance obtained from Irons et al. (1992).

\section{RESULTS}

It should be noted that the red band is sensitive to atmospheric scattering and plant absorption, while the NIR band is very sensitive to the amount of vegetation (scattering) and atmospheric absorption. As vegetation cover increases an asymptotic relationship with fAPAR is commonly seen, while LAI continues to increase as the vegetation develops. This is similar to the behavior of red and NIR, respectively. Thus, saturation varies with different bands and vegetation physical parameters. This has to be considered when evaluating the sensitivity of VI's to different vegetation parameters.

The analysis carried out here should take into account the effect of the interaction between ground cover (GC) and clump leaf area index (CLAI), implicit in the Myneni's model (Fig. la-c). For the same LAI (GC $x$ CLAI) it is possible to have different combinations of CLAl and GC (e.g. for $L A I=1$ we can have two conditions: $\mathrm{CLAl}=4$ and $\mathrm{GC}=0.25$, and $\mathrm{CLAI}=2$ and $\mathrm{GC}=0.5$ ). When the LAI $v s$. VI relationships are plotted, the points where $\mathrm{LAI}=1$ and 2 , are clearly affected by this interaction. In these points the lower ground cover $(0.25$ and 0.50$)$ associated with CLAI $=4$ depresses the VI values, irrespective of the soil brightness and view angle. This is due to the coupled effect of increasing the red and decreasing the NIR reflectance with the exposure of more soil surface and less vegetation, causing lower VI values. This makes the interpretation of spectral indices values in relation to physical vegetation parameters more difficult. Thus, for the same view and illumination geometries, same soil and atmosphere conditions, and same vegetation parameter (e.g. LAI) different spectral relationships over different ground cover geometries will be found. This is clear in the discussion that follows.

The relationship between VI's and LAI are shown in Figs. la-c for NDVI and SAVI. The NDVI shows an asymptotic 
behavior as LAI increases, mainly if we decouple the CLAI/GC effect; for the SAVI this is less visible, and the relationship is more linear. The view angle variations in the principal plane $\left(-60^{\circ}\right.$ to $+60^{\circ}$ ) cause the NDVI and SAVI to vary from less than $20 \%$ for high LAI values (Fig. la) to more than $100 \%$ if we consider the GC/CLAI effect altogether (e.g. LAI $=1$ ) and more than $30 \%$ if we decouple the GC/CLAI effect. For the SR the variations can be as high as $200 \%$ (results not shown). When there is an atmosphere between the target and the sensor, the asymptotic behavior of NDVI is less stressed (Fig. 1b); the SAVI (Fig. 1c) almost doesn't change its behavior. The atmosphere causes the dynamic range of all indices to decrease, mainly because the coupled effect of the increase in the red (scattering) and decrease in the NIR (absorption). With the atmosphere the GC/CLAI effect is more clearly seen (Figs. $1 \mathrm{a}, \mathrm{b})$, as there is a clear separation between the groups of $\mathrm{CLAI}=2$ and $\mathrm{CLAI}=4$ (observe the points $\mathrm{LAI}=1$ and $\mathrm{LAI}=2$ ). There is a coupled effect of atmosphere and LAI on the NDVI (Fig. la,b). When there is no atmosphere, the view angle significantly alters the magnitude of the NDVI since the signal in the red band is low (the contribution of the atmosphere to red scattering is absent) and the changes in red caused by illuminated/shadowed views at lower LAI's make the NDVI change significantly. For higher LAI's, changes in red due to view angle variations are smaller and consequently the NDVI changes less as a function of view angle. However, when the atmosphere is present (high red contribution), there is an inverse effect between view angle effect and atmospheric effect on the NDVI. When the view angle departs from nadir (mainly towards the forward scattering direction) the signal coming from the canopy in the red band decreases; however. the contribution from the atmosphere increases, thus stabilizing the NDVI. For higher LAI values, changes in red canopy reflectance itself caused by changes in view angle are not as high as those caused by atmospheric effects, thus introducing variations in NDVI. Generally, as the view angles depart from the nadir the red increases in response to increases in the path radiance, thus tightening the VI values and restricting large view angle variations, mainly for low LAI conditions.

The relationships between AAPAR and NDVI and SAVI in the absence of an atmosphere and for a constant soil red reflectance of 0.20 are shown in Figs. 2a-b. The relationships are slightly more linear for SAVI than for NDVI. The variations in the fAPAR axis are mainly due to variations in ground cover and not due to CLAI. The LAI variations exert a small influence on fAPAR, which reinforce the observations that fAPAR is highly correlated with canopy capability for light interception (ground cover). For a given ground cover, loadings in CLAI cause small increments in fAPAR. However, coupled changes in LAI and view angle cause the VI's to change. For a given fAPAR value, the SAVI (Fig. $2 b$ ) tends to show less view angle variations and lower variations due to clump LAI for a constant view angle than NDVI. The NDVI tends to show lower values for the hot spot $\left(-40^{\circ}\right)$, while the SAVI shows the highest values at this specific view angle. The displacement in SAVI $v$ s. fAPAR relationship due to view angle variations are more parallell than for NDVI, suggesting that an offset correction could work better in the SAVI than in the NDVI.

The atmosphere loading caused the view angle curves to get closer (Fig. lc), as a result of increased red scattering, mainly when view angles depart from nadir. The atmosphere seems to function as a buffer against view angle effects in relationships between VI and fAPAR. Nevertheless, the atmosphere changes the inclination and intercept of these relationships. And different atmospheric loadings will cause different relationships.

A part from the impact that view angle, soil variation, or atmospheric loading has on the VI, it is interesting to evaluate the resulting impact estimating vegetation parameters. Figures $3 a, b$ summarize linear regression coefficients between NDVI and SAVI and AAPAR as a function of view angle and soil reflectance variation. The SAVI shows higher linear regression coefficients for all view angles than the NDVI, irrespective of the soil or atmospheric condition. This can be attributed to the soil brightness correction present in the SAVI formulation. For both indices, the effects of view angle and soil brightness are more pronounced when atmosphere is not present. When there is an atmospheric loading, the red values become higher and consequently the NDVI becomes less sensitive to changes in red values (soil effects become lower). Generally, as the red values decrease. there is an increase in sensitivity of the VI's, thus disturbing the relationship with fAPAR.
The worst view angles are the extremes $\left( \pm 70^{\circ}\right)$ and the hot spot (backscattering and forward scattering) view angles $\left( \pm 40^{\circ}\right)$. However, for the NDVI and in the absence of atmosphere, the range of best view angles is reduced. It is interesting to observe that while the hot spot causes high radiance values, the correlation between fAPAR and NDVI or SAVI is lower for this specific sun-view configuration. Thus, the hot spot actually worsens VI-physical parameter relationships.

\section{CONCLUSIONS}

The interaction GC/CLAI affected the relationship between VI and LAI to a greater extent than FAPAR, because the fAPAR was much more a function of the ground cover than the amount of vegetation itself. Neverthess, different LAI's of the clumps create "families" of relationships between VI and vegetation parameters. Thus, in large scale vegetation monitoring, it is important to stratify the vegetation according their CLAI/GC characteristics. The relationship between LAI and SAVI was more linear than with NDVI, suggesting a higher sensitivity of the SAVI to NIR than the NDVI. Both indices showed linear relationships with fAPAR; however, the regression coefficients for SAVI were higher than those for NDVI, for all view angles studied and in presence and absence of atmosphere. For both indices the hot spot and extreme view angles were more restrictive for relationships with fAPAR.

\section{ACKNOWLEDGEMENTS}

The former author is supported by National Council for Scientific Development (CNPq/Brazil).

\section{REFERENCES}

Daughtry, C.S.; Gallo, K.P.; Goward. S.N.; Prince, S.D.; Kustas, W.P. "Spectral estimates of absorbed radiation and phytomass production in corn and soybean canopies." Remote Sens. Environ. 39(1992):141-152

Goward, S.N.; Huemmrich, K.F. "Vegetation canopy PAR absorptance and the normalized difference vegetation index: an assessment using the SAIL model." Remote Sens. Environ. 39(1992):119-140.

Huete, A.R. "A soil-adjusted vegetation index (SAVI)." Remote Sens. Environ. 25(1988):295-309.

Irons, J.R.; Campbell, G.S.; Norman, J.M.; Graham, D.W.; Kovalick, W.M. "Prediction and measurement of soil bidirectional reflectance." IEEE Trans. Geosci. Remote Sens. 30(1992):249-260.

Jacquemoud, S.; Baret, F. "Prospect: a model of leaf optical properties spectra." Remote Sens. Environ. 34(1990):75-91.

Kaufman, Y.J.; Tanré, D. "Atmospherically resistant vegetation index (ARVI) for EOS-MODIS." IEEE Trans. Geosci. Remote Sens. 30(1992):261-270.

Myneni, R.M.: Asrar, G.: Hall F.G. "A three-dimensional radiative transfer method for optical remote sensing of vegetated land surfaces." Remote Sens. Environ. 41(1992b): 105-121. 

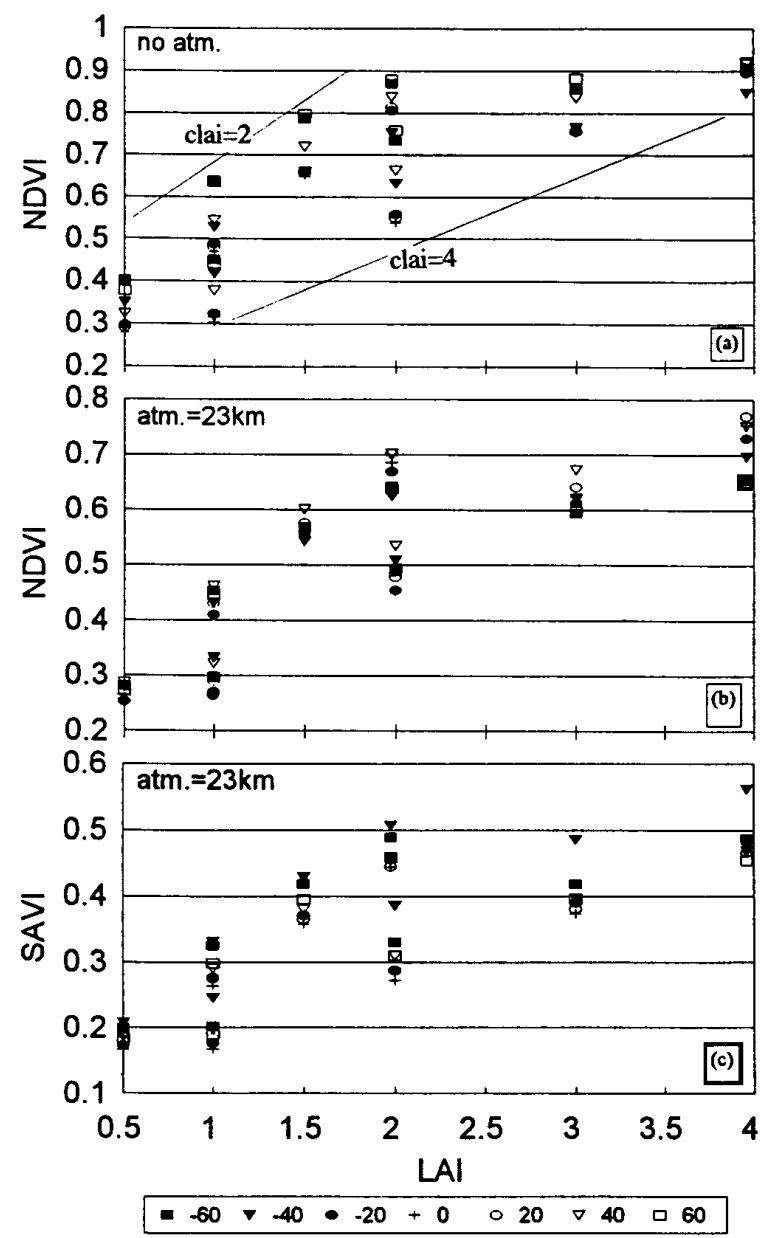

Figure 1 - Relationships between LAI and NDVI (a,b) and SAVI (c), for different view angles; soil red reflectance is 0.20 .
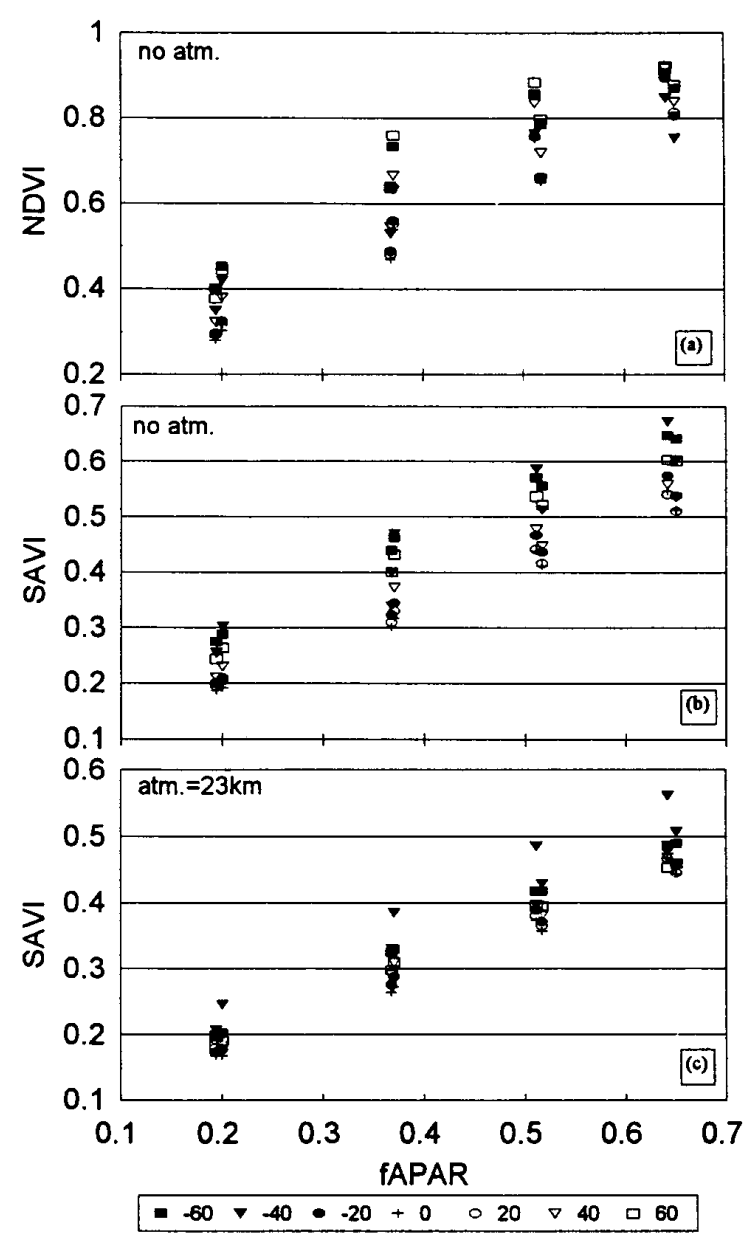

Figure 2 - Relationships between fAPAR and NDVI (a) and SAVI $(b, c)$, for different view angles; soil red reflectance is 0.20 .
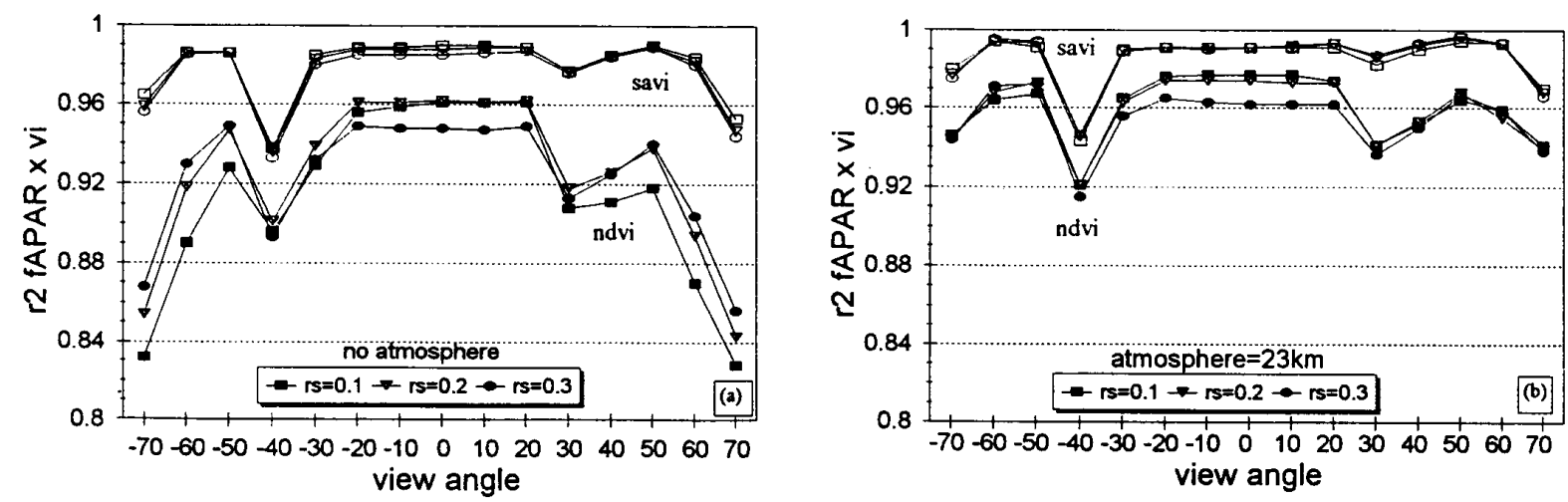

Figure 3 - Variation of the linear regression coefficients between fAPAR and NDVI and SAVI along the view angles, for three soil reflectances, and in the absence (a) and presence of atmosphere (b). 